International Electronic Journal of Algebra

VOLUMe 21 (2017) 121-126

\title{
CENTRAL TORSION UNITS OF INTEGRAL REALITY-BASED ALGEBRAS WITH A POSITIVE DEGREE MAP
}

\author{
Allen Herman and Gurmail Singh \\ Received: 22 April 2016; Revised 2 September 2016 \\ Communicated by Abdullah Harmancı
}

\begin{abstract}
A reality-based algebra (RBA) is a finite-dimensional associative algebra that has a distinguished basis $\mathbf{B}$ containing $1_{A}$, where $1_{A}$ is the identity element of $A$, that is closed under a pseudo-inverse condition. If the RBA has a one-dimensional representation taking positive values on $\mathbf{B}$, then we say that the RBA has a positive degree map. When the structure constants relative to a standardized basis of an RBA with positive degree map are all integers, we say that the RBA is integral. Group algebras of finite groups are examples of integral RBAs with a positive degree map, and so it is natural to ask if properties known to hold for group algebras also hold for integral RBAs with positive degree map. In this article we show that every central torsion unit of an integral RBA with algebraic integer coefficients is a trivial unit of the form $\zeta b$, for some $\zeta$ is a root of unit in $\mathbb{C}$ and $b$ is an element of degree 1 in $\mathbf{B}$.
\end{abstract}

Mathematics Subject Classification (2010): 16U60, 05E30, 16S99, 20N99 Keywords: Reality based algebra, table algebra, association schemes, unit group, torsion units

\section{Introduction}

A reality based algebra $(\mathrm{RBA})$ is a pair $(A, \mathbf{B})$, where $A$ is a finite-dimensional algebra over $\mathbb{C}$ with involution $*$ and has a distinguished basis $\mathbf{B}=\left\{b_{0}, b_{1}, \ldots, b_{d}\right\}$ for which:

(i) the involution $*: A \rightarrow A$ is $\mathbb{R}$-linear and $\mathbb{C}$-conjugate linear and satisfies $(u v)^{*}=v^{*} u^{*}$, for all $u, v \in A$;

(ii) $b_{0}=1_{A} \in \mathbf{B}$;

(iii) $\left(b_{i}\right)^{*}=b_{i^{*}} \in \mathbf{B}$, for all $b_{i} \in \mathbf{B}$, for a transposition $*:\{0,1, \ldots, d\} \rightarrow$ $\{0,1, \ldots, d\}$ 
(iv) multiplication in $A$ defines real structure constants in the basis $\mathbf{B}$, i.e. for all $b_{i}, b_{j} \in \mathbf{B}$, we have

$$
b_{i} b_{j}=\sum_{b_{k} \in \mathbf{B}} \lambda_{i j k} b_{k}, \text { for some } \lambda_{i j k} \in \mathbb{R}
$$

(v) for all $b_{i}, b_{j} \in \mathbf{B}, \lambda_{i j 0} \neq 0 \Longleftrightarrow j=i^{*}$; and

(vi) for all $b_{i} \in \mathbf{B}, \lambda_{i i^{*} 0}=\lambda_{i^{*} i 0}>0$.

Reality-based algebras were introduced by Blau in [1] to provide a bridge between adjacency algebras of association schemes, table algebras, the $C$-algebras of Kawada, fusion rule algebras, and several other kinds of discrete hypergroups.

When the map $\delta: A \rightarrow \mathbb{C}$ given by $\delta(\alpha)=\sum_{i} \alpha_{i} \delta\left(b_{i}\right)$ is an algebra $*$-homomorphism of $A$ that takes positive values on elements of the basis $\mathbf{B}$, then we say that $\delta$ is a positive degree map for the $\operatorname{RBA}(A, \mathbf{B})$. The degree map is unique to the RBA, and the distinguished basis can be rescaled to a standardized RBA-basis that has $\delta\left(b_{i}\right)=\lambda_{i i^{*} 0}$ for all $i=0, \ldots, d$. If, in addition, the structure constants $\lambda_{i j k}$ with respect to a standardized RBA-basis are all integers (resp. rationals), then we say that the $\operatorname{RBA}(A, \mathbf{B})$ is an integral (resp. rational) RBA with positive degree map.

The prototypical example of an integral RBA with positive degree map is the complex group algebra $\mathbb{C} G$ of a finite group $G$, whose distinguished RBA-basis $G$ is a standardized RBA-basis for the degree map given by the augmentation map (or the trivial character) having $\delta(g)=1$ for all $g \in G$. Bose-Mesner algebras of finite association schemes and integral table algebras give other useful and interesting cases of integral RBAs with positive degree map.

For an integral RBA $(A, \mathbf{B})$, the fact that the structure constants are integral means that $\mathbb{Z} \mathbf{B}$ is a subring of $A$. An important theme in integral representation theory is to study the groups of units of $\mathbb{Z}$-orders of semisimple algebras over $\mathbb{C}$. In this article we study torsion units (i.e. units of finite order) in $\mathbb{Z} \mathbf{B}$, when $(A, \mathbf{B})$ is an integral RBA with positive degree map. It is easy to see in this case that the elements of $\mathbf{B}$ of degree 1 form a finite subgroup of $\mathbf{B}$, which we denote by $L(B)$. The units of $A$ having the form $\zeta b_{i}$, where $\zeta \in \mathbb{C}^{\times}$and $b_{i} \in L(B)$, are called trivial units of $A$. The main result of this article shows that central torsion units of $\mathbb{Z} \mathbf{B}$ must be trivial units whenever $(A, \mathbf{B})$ is an integral RBA with positive degree map. Motivated by the Zassenhaus conjecture for integral group rings of finite groups, we ask if every torsion unit of $\mathbb{Z} \mathbf{B}$ will be conjugate in $A$ to a trivial torsion unit when $(A, \mathbf{B})$ is an integral RBA with positive degree map. 


\section{2. $C^{*}$-algebra representation theory for RBAs}

In this section our goal is to show that every $\operatorname{RBA}(A, \mathbf{B})$ has a faithful *representation $\Gamma: A \rightarrow M_{n}(\mathbb{C})$ for which $\Gamma\left(u^{*}\right)=\overline{\Gamma(u)}^{\top}$ for all $u \in A$. This is a consequence of the representation theory of finite-dimensional semisimple $C^{*}$ algebras. First we establish that RBAs are semisimple algebras. This was observed previously in [1, Proposition 2.3]. The easy proof presented here is from [4].

Proposition 2.1. Let $(A, \mathbf{B})$ be an $R B A$. Then $A$ is a finite-dimensional semisimple algebra.

Proof. $A$ is a finite-dimensional algebra, so its radical $J$ is nilpotent. Since the involution $*$ is an antiautomorphism, $J^{*}=J$. Let $m$ be the least positive integer for which $J^{m}=0$. Then $I=J^{m-1}$ is a nonzero ideal of $A$ with $I^{2}=0$. If $x=\sum_{i} x_{i} b_{i}$ is a nonzero element of $I$, then $x^{*} \in I$, so $x^{*} x=0$. But the coefficient of $b_{0}$ in $x^{*} x$ is $\sum_{i}\left|x_{i}\right|^{2} \lambda_{i i^{*} 0}>0$, a contradiction. Therefore, $J=0$, so $A$ is semisimple.

If $u \in A, u_{0}$ denotes the coefficient of $b_{0}$ in the expression of $u$ in terms of the basis $\mathbf{B}$. Every $\operatorname{RBA}(A, \mathbf{B})$ has a feasible trace $u \mapsto u_{0}$; this is $*$-linear on $A$ and satisfies $\left(x y^{*}\right)_{0}=\left(y x^{*}\right)_{0}$. This implies that

$$
\langle x, y\rangle=\left(x y^{*}\right)_{0}, \quad \text { for all } x, y \in A,
$$

is a nondegenerate hermitian form on $A$. In particular, $\|x\|=\sqrt{\langle x, x\rangle}$ defines a norm on $A$. Being a finite-dimensional vector space over $\mathbb{C}, A$ is complete with respect to this norm, so $A$ is a $C^{*}$-algebra.

Now that we have established that our RBA $A$ is a finite-dimensional semisimple $C^{*}$-algebra, we can apply the construction of $[5, \S 11]$ to obtain a regular *representation. First, every simple component of the Wedderburn decomposition $A=\oplus_{\chi} A e_{\chi}$ is a two-sided $*$-ideal of $A$, as $\chi$ runs through the distinct irreducible characters of $A$. Each of these simple components $A e_{\chi}$ is a simple finitedimensional $C^{*}$-algebra. The construction of [5, Theorem 11.2] produces a faithful *-representation $\Gamma_{\chi}: A e_{\chi} \rightarrow M_{n_{\chi}}(\mathbb{C})$ for every simple component of $A$, where $n_{\chi}=\operatorname{tr}\left(\Gamma_{\chi}\left(e_{\chi}\right)\right)$ for all $\chi \in \operatorname{Irr}(A)$. Each $\Gamma_{\chi}$ lifts to a representation $\hat{\Gamma}_{\chi}$ of $A$, and taking the direct sum of these representations produces a faithful $*$-representation

$$
\Gamma: A=\oplus_{\chi} A e_{\chi} \rightarrow \oplus_{\chi} M_{n_{\chi}}(\mathbb{C}) \hookrightarrow M_{n}(\mathbb{C}),
$$

where $n=\sum_{\chi} n_{\chi}$.

The next proposition shows how we can apply a faithful $*$-representation to torsion units of an RBA. 
Proposition 2.2. Let $(A, \mathbf{B})$ be an $R B A$. Then every torsion unit of $A$ is conjugate in $A$ to $a$ *-unitary torsion element of $A$.

Proof. Let $\Gamma$ be the faithful *-representation of $A$ as constructed above. When $u \in A$ is a torsion unit of multiplicative order $k$, then $\Gamma(u)$ is a torsion matrix of order $k$. This means each block of $\Gamma(u)$ is a torsion matrix, and hence diagonalizable. Let $W_{\chi}$ be invertible $n_{\chi} \times n_{\chi}$ matrices for which $W_{\chi}^{-1} \Gamma_{\chi}\left(u e_{\chi}\right) W_{\chi}$ is diagonal, for all $\chi \in \operatorname{Irr}(A)$. Since each block constituent representation $\Gamma_{\chi}: A \rightarrow M_{n_{\chi}}(\mathbb{C})$ is irreducible, it is also surjective. Let $w e_{\chi} \in A e_{\chi}$ with $\Gamma_{\chi}\left(w e_{\chi}\right)=W_{\chi}$, and set $w=\sum_{\chi} w e_{\chi}$. Then $\Gamma\left(w^{-1} u w\right)$ is a diagonal torsion matrix, and hence $*$-unitary:

$$
\Gamma\left(w^{-1} u^{-1} w\right)=\Gamma\left(w^{-1} u w\right)^{-1}={\overline{\Gamma\left(w^{-1} u w\right)}}^{\top}=\Gamma\left(\left(w^{-1} u w\right)^{*}\right) .
$$

Since $\Gamma$ is a faithful $*$-isomorphism, this proves $\left(w^{-1} u w\right)^{-1}=\left(w^{-1} u w\right)^{*}$, as required.

\section{Torsion units for RBAs with positive degree map}

Suppose now that the RBA $(A, \mathbf{B})$ has a positive degree map $\delta$. When the RBAbasis $\mathbf{B}$ is standardized with respect to $\delta$, we can define the order of the RBA to be

$$
n:=\delta\left(\mathbf{B}^{+}\right)=\sum_{i=0}^{d} \delta\left(b_{i}\right) .
$$

The positive degree map also puts us in a position to define the standard feasible trace $\rho: A \rightarrow \mathbb{C}$ with $\rho(\alpha)=n \alpha_{0}$ for all $\alpha=\sum_{i} \alpha_{i} b_{i} \in A$. Being a nonsingular trace function on the finite-dimensional semisimple algebra $A, \rho$ can be expressed as a linear combination of the irreducible characters of $A[3, \S 5]$. The coefficients $m_{\chi}$ in this linear combination $\rho=\sum_{\chi} m_{\chi} \chi$ are the multiplicities of the RBA. Higman's character formula $[3]$

$$
e_{\chi}=\frac{m_{\chi}}{n} \sum_{i} \frac{\chi\left(b_{i}^{*}\right)}{\delta\left(b_{i}\right)} b_{i}
$$

tells us how to express the centrally primitive idempotents of $A$ in terms of the standardized basis $\mathbf{B}$. Since $\chi\left(b_{i^{*}}\right)=\overline{\chi\left(b_{i}\right)}$ for all $b_{i} \in \mathbf{B}$, we have

$$
\chi\left(e_{\chi}\right)=\chi\left(b_{0}\right)=\frac{m_{\chi}}{n} \sum_{i} \frac{\overline{\chi\left(b_{i}\right)} \chi\left(b_{i}\right)}{\delta\left(b_{i}\right)} .
$$

$\chi\left(b_{0}\right)=n_{\chi}$ is the degree of $\chi$ so it is always a positive integer. When the degrees $\delta\left(b_{i}\right)$ are all positive and real, then $n$ is positive and real, so the multipicity $m_{\chi}$ is positive and real. It follows then that $e_{\chi}^{*}=e_{\chi}$. (Note that this implies that 
every two-sided ideal of $A$ is $*$-invariant, in agreement with the finite-dimensional $C^{*}$-algebra theory.)

The next lemma was proved in [2], and in [6] for commutative RBAs with positive degree map. We include a proof here for completeness.

Lemma 3.1. Let $(A, \mathbf{B})$ be a rational $R B A$ with positive degree map and suppose $B$ is a standardized RBA-basis. Suppose the degrees of $B$ satisfy $\delta\left(b_{i}\right) \geq 1$ for all $i$. Then the only $*$-unitary units of $A$ with totally real algebraic integer coefficients are the trivial units of the form $\pm b_{i}$ for some $b_{i} \in L(B)$.

Proof. Let $u=\sum_{i} u_{i} b_{i}$ be a $*$-unitary unit with coefficients $u_{i} \in \overline{\mathbb{Z}}$ for all $i$. Then $b_{0}=u u^{*}$ implies that $1=\sum_{i}\left|u_{i}\right|^{2} \delta\left(b_{i}\right)$. Since the $\left|u_{i}\right|^{2}$ are nonnegative real and all of the $\delta\left(b_{i}\right)$ are $\geq 1$, we have that $\left|u_{i}\right|^{2} \leq 1$ for all $i$.

Let $\overline{\mathbb{Q}}$ denote an algebraic closure of $\mathbb{Q}$ in $\mathbb{C}$. Since the structure constants in the basis $\mathbf{B}$ are all rational, the action of any Galois automorphism $\sigma \in \operatorname{Gal}(\overline{\mathbb{Q}} / \mathbb{Q})$ on coefficients yields an automorphism of the algebra $A$. If $u_{i}$ is a totally real algebraic integer, then $\sigma\left(\overline{u_{i}}\right)=\sigma\left(u_{i}\right)=\overline{\sigma\left(u_{i}\right)}$ for all $i$, and hence $\sigma\left(u u^{*}\right)=\sigma(u) \sigma(u)^{*}$. In particular, $\sigma(u)$ is also a $*$-unitary unit of $A$. Therefore, for every $i$ we have that $\left|\sigma\left(u_{i}\right)\right|^{2} \leq 1$ for all $\sigma \in \operatorname{Gal}(\overline{\mathbb{Q}} / \mathbb{Q})$, and so by a classical result of Kronecker, every $u_{i}$ is either 0 or a root of unity. Since $u \neq 0,1=\sum_{i}\left|u_{i}\right|^{2} \delta\left(b_{i}\right)$, and $\delta\left(b_{i}\right) \geq 1$ for all $i$, it follows that there is a unique $i$ for which $u_{i}$ is a root of unity, and for this $i$ the degree of $b_{i}$ is 1 . Since the basis $\mathbf{B}$ is standardized with respect to the positive degree map $\delta$, this impies $b_{i} b_{i}^{*}=b_{0}$, so $u=u_{i} b_{i}$ is a trivial unit. Since $u_{i}$ is also totally real, it must be the case that $u= \pm b_{i}$.

Theorem 3.2. Let $(A, \mathbf{B})$ be a rational $R B A$ with positive degree map $\delta$ and standardized RBA-basis $\mathbf{B}$. Assume that $\delta\left(b_{i}\right) \geq 1$ for all $b_{i} \in \mathbf{B}$. Then every central torsion unit of $A$ with algebraic integer coefficients is a trivial unit.

Proof. If $u$ is a central torsion unit with $u^{k}=b_{0}$, then $u=\sum_{\chi} u_{\chi} e_{\chi}$ is a $\mathbb{C}$-linear combination of the centrally primitive idempotents of $A$, and the coefficients $u_{\chi}$ are all $k$-th roots of unity. In particular, we have $\overline{u_{i}}=u_{i}^{-1}$ for all $i$, and so $u^{*}=u^{-1}$. If $\sigma \in \operatorname{Gal}(\overline{\mathbb{Q}} / \mathbb{Q})$, then every $\sigma\left(u_{i}\right)$ is a $k$-th root of unity, so

$$
\sigma\left(u^{*}\right)=\sum_{\chi} \sigma\left(u_{i}\right)^{-1} \sigma\left(e_{\chi}\right)=\sum_{\chi} \overline{\sigma\left(u_{i}\right)} \sigma\left(e_{\chi}\right)=\sigma(u)^{*} .
$$

As before it follows that $1=\sum_{i}\left|\sigma\left(u_{i}\right)\right|^{2} \delta\left(b_{i}\right)$ for all $\sigma \in \operatorname{Gal}(\overline{\mathbb{Q}} / \mathbb{Q})$. If all of the $u_{i}$ are algebraic integers, then this implies as before that all of the $u_{i}$ are either 0 or roots of unity, and it again must be the case that there is a unique nonzero $u_{i}$ for 
which $\delta\left(b_{i}\right)=1$. In this case $u=u_{i} b_{i}$ where $u_{i}$ is a root of unity and $b_{i} \in L(B)$ is central in $A$.

Corollary 3.3. If $(A, \mathbf{B})$ is an integral $R B A$ with positive degree map, then every central torsion unit of $\mathbb{Z} \mathbf{B}$ is a trivial unit.

We can ask if the Zassenhaus conjecture for integral group rings holds in the more general setting of integral RBAs with positive degree map. This is the statement that every torsion unit of $\mathbb{Z} \mathbf{B}$ is conjugate in $\mathbb{C B}$ to a trivial unit. Corollary 3.3 is a necessary step in this direction. In fact the only torsion units of $\mathbb{Z} \mathbf{B}$ that are guaranteed to exist are the trivial units and their conjugates, so the problem is to show these are the only torsion units present. The Zassenhaus conjecture has been studied extensively for integral group rings of finite groups. For an up-to-date account, see [4].

\title{
References
}

[1] H. I. Blau, Table algebras, European J. Combin., 30(6) (2009), 1426-1455.

[2] A. Herman and G. Singh, On the torsion units of integral adjacency algebras of finite association schemes, Algebra, 2014 (2014), Article ID 842378, 5 pages.

[3] D. G. Higman, Coherent algebras, Linear Algebra Appl., 93 (1987), 209-239.

[4] G. Singh, Torsion Units of Integral Group Rings and Scheme Rings, Ph.D. Thesis, University of Regina, 2015.

[5] M. Takesaki, Theory of Operator Algebras I, Encyclopaedia of Mathematical Sciences, 124, Springer-Verlag, Berlin, 1979.

[6] B. Xu, On isomorphisms between integral table algebras and applications to finite groups and association schemes, Comm. Algebra, 42(12) (2014), 52495263.

\author{
Allen Herman (Corresponding Author) and Gurmail Singh \\ Department of Mathematics and Statistics \\ University of Regina \\ Regina, Canada, S4S 0A2 \\ e-mails: Allen.Herman@uregina.ca (A. Herman) \\ singh28g@uregina.ca (G. Singh)
}

